\title{
A new approach to film dosimetry for high energy photon beams: Lateral scatter filtering
}

\author{
Sandra E. Burch \\ Department of Radiology, Medical College of Georgia, Augusta, Georgia 30912 \\ Kimberlee J. Kearfott \\ Department of Nuclear Engineering, University of Michigan, Ann Arbor, Michigan 48109 \\ Jon H. Trueblood and W. Chris Sheils \\ Department of Radiology, Medical College of Georgia, Augusta, Georgia 30912 \\ J. Inhwan Yeo and C. K. Chris Wang \\ School of Mechanical Engineering, Georgia Institute of Technology, Atlanta, Georgia 30332
}

(Received 21 August 1996; accepted for publication 25 February 1997)

\begin{abstract}
A method of film dosimetry for high energy photon beams is proposed which reduces the required film calibration exposures to a set of films obtained for a small radiation field size and shallow depth $(6 \mathrm{~cm} \times 6 \mathrm{~cm}$ at $5 \mathrm{~cm}$ depth). It involves modification of a compression type polystyrene film phantom to include thin lead foils parallel to the vertical film plane at approximately $1 \mathrm{~cm}$ from both sides of the film emulsion. The foils act as high atomic number filters which remove low energy Compton scatter photons that otherwise would cause the film sensitivity to change with field size and depth. The proposed method is best described as "lateral scatter filtering." To validate the proposed method, central axis depth doses and isodose curves for a 4 MV photon beam were determined from films exposed within the modified phantom and the results compared with ionization chamber measurements. When no lateral filtering was used, for field sizes of $6 \mathrm{~cm} \times 6 \mathrm{~cm}$ and $25 \mathrm{~cm} \times 25 \mathrm{~cm}$, this comparison demonstrated up to a $65 \%$ difference between film and ionization chamber central axis depth dose measurements. When using the lateral scatter filtering technique, less than a 4\% difference was observed for these field sizes. (C) 1997 American Association of Physicists in Medicine. [S0094-2405(97)01805-1]
\end{abstract}

Key words: film dosimetry, radiation therapy, phantoms, photon dose measurement

\section{INTRODUCTION}

Radiation therapy dosimetric studies using radiographic film have been performed since the introduction of cobalt- 60 teletherapy and high energy betatrons for clinical use. ${ }^{1}$ The advantages of film over other measurement techniques include speed of data collection, low cost, improved spatial resolution, and simultaneous integration of dose at all data points. Film is potentially the ideal detector for determining dose distributions for dynamic beams and for studying combinations of stationary beams treated sequentially (e.g., gap dosimetry). Both of these situations are difficult to measure using conventional water phantom dosimetry systems, since the dose distribution changes with time.

Although film dosimetry is frequently used to determine relative dose distribution for electron beam therapy, measurement of dose distribution for photon beam therapy is not widely accepted. This low level of acceptance is a result of the fact that the film sensitivity varies as the distribution of photon energies shifts within a tissue equivalent phantom with field size and depth. ${ }^{2}$ Relative to ion chamber measurements, differences of $30 \%$ or more in percentage depth dose values have been observed for a cobalt $6010 \mathrm{~cm} \times 10 \mathrm{~cm}$ field at depths greater than $15 \mathrm{~cm}^{3}$ Differences up to $5 \%$ for $25 \mathrm{MV}$ accelerator beams have been reported. ${ }^{4}$

High energy photon beams used in radiation oncology are considered to interact primarily by Compton scattering processes with tissue. However, when film is placed in a tissue equivalent material, photoelectric interactions associated with the silver atoms in the emulsion cause the film to overrespond relative to the tissue equivalent material. Since the probability for photoelectric interaction is proportional to the third power of the atomic number, this causes significant inaccuracies in film dosimetry even for high energy beams. ${ }^{3}$ The problem occurs because of the presence of photons with energies below $400 \mathrm{keV}$ for which the photoelectric effect is significant. For the lower energy photons, film dose may be as much as 25 times the tissue dose at the same physical location. ${ }^{5}$ The shift in the energy spectrum of the scattered beam with depth in tissue is different than for the primary beam because there is an increase in singly and multiply scattered photons with the increase in depth and also with field size. According to the equations for Compton scattering, the energy of the scattered photon is related to the incident photon energy as follows:

$$
h \nu^{\prime}=h \nu \cdot \frac{1}{1+\alpha(1-\cos \phi)},
$$

where $h \nu$ is the energy of the incident photon, $h \nu^{\prime}$ is the scattered photon energy, $\phi$ is the angle at which the scattered photon emerges and $m_{0} c^{2}$ is the rest mass energy of the electron, 


$$
\alpha=\frac{h \nu}{m_{0} c^{2}}=\frac{h \nu \mathrm{MeV}}{0.511 \mathrm{MeV}}
$$

For photons scattered at $90^{\circ}$, the equation reduces to

$$
h \nu^{\prime}=\frac{h \nu}{1+\alpha}
$$

since the cosine of $90^{\circ}$ is zero. An incident photon with an energy of $4 \mathrm{MeV}$ will produce a $90^{\circ}$ scattered photon of energy $0.453 \mathrm{MeV}$ and for a $4 \mathrm{MeV}$ incident photon scattered at $180^{\circ}$, the energy is $0.240 \mathrm{MeV}$. It is clear that the beam energy is quickly degraded for large angle scatter and that for multiply scattered photons the energy would be low enough to produce the observed over-response.

Various methods have been used to correct photon film data to obtain acceptable results. For example, one method uses ion chamber central axis data in combination with offaxis data from film ${ }^{6}$ while another method uses a curve fitting approach based on one reference field size to provide correction for variation of film sensitivity with depth. ${ }^{3}$ Alternatively, to perform photon film dosimetry for dynamic wedged fields one can measure film calibration curves for a range of field sizes and depths to obtain correction factors, which may require exposing over 200 calibration films for a complete evaluation.

The method of film dosimetry proposed here attempts to limit the required film calibration exposures to a single set of films (8-10) for a small field size and shallow depth $(6 \mathrm{~cm} \times 6 \mathrm{~cm}$ at $5 \mathrm{~cm}$ depth). The fraction of the dose to the phantom due to low energy photons should be lowest for these conditions (i.e., less scatter for smaller fields). This method involves modification of a polystyrene compressed sandwich type phantom used for film irradiation to include sheets of lead foil parallel to the film plane. The lead foils are placed at distances slightly greater than $1 \mathrm{~cm}$ from both sides of the emulsion and serve as high atomic number filters to selectively eliminate the low energy lateral scatter which is responsible for the increase in film sensitivity with field size and depth (Fig. 1). A single phantom configuration with fixed foil thickness and distance is used for all field sizes for a given photon beam energy, although a different configuration may be needed for other beam energies. The primary objective is to remove as many of the low energy photons as possible without appreciably altering the dose distribution to the phantom within this plane. The lead foil filters result in some attenuation of the the entire spectrum at all depths and this limits the accuracy which can be obtained using the proposed method unless central axis depth doses are corrected using ion chamber data.

Monte Carlo technique (ITS code ${ }^{7}$ ) was used to demonstrate the change in the photon spectra reaching the film plane at various depths. Figure 2 shows that on the central ray, at depths of 5.4 and $18.4 \mathrm{~cm}$, the number of photons with energies greater than $400 \mathrm{keV}$ is almost the same with or without the filters in place. However, the number of photons reaching the film with energies below $400 \mathrm{keV}$ is greatly reduced. These low energy photons account for only a small fraction of the dose to tissue, because there are few

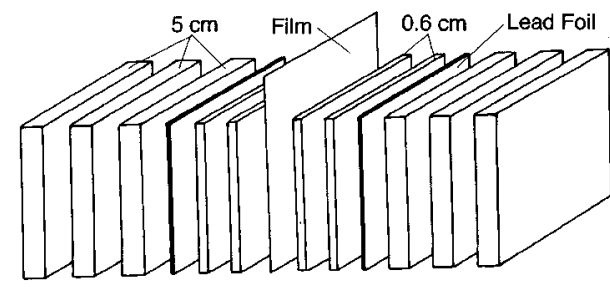

Polystyrene Phantom

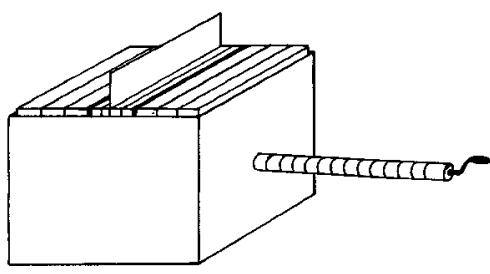

Phantom Compression Device

FIG. 1. The polystyrene film phantom with removable lead foil inserts. For vertical exposure parallel to the beam direction, the film is compressed within the polystyrene by the aluminum compression device. For film exposure perpendicular to the beam direction, the lead foils are removed and the polystyrene sheets are stacked on the table without the compression device.

of them and each carries relatively little energy. Even so, they impart a much higher dose to film than to tissue and their removal should dramatically decrease the over-response of film.

The method does not require extensive data collection and involves no mathematical manipulation other than the straightforward conversion of density to dose based on the

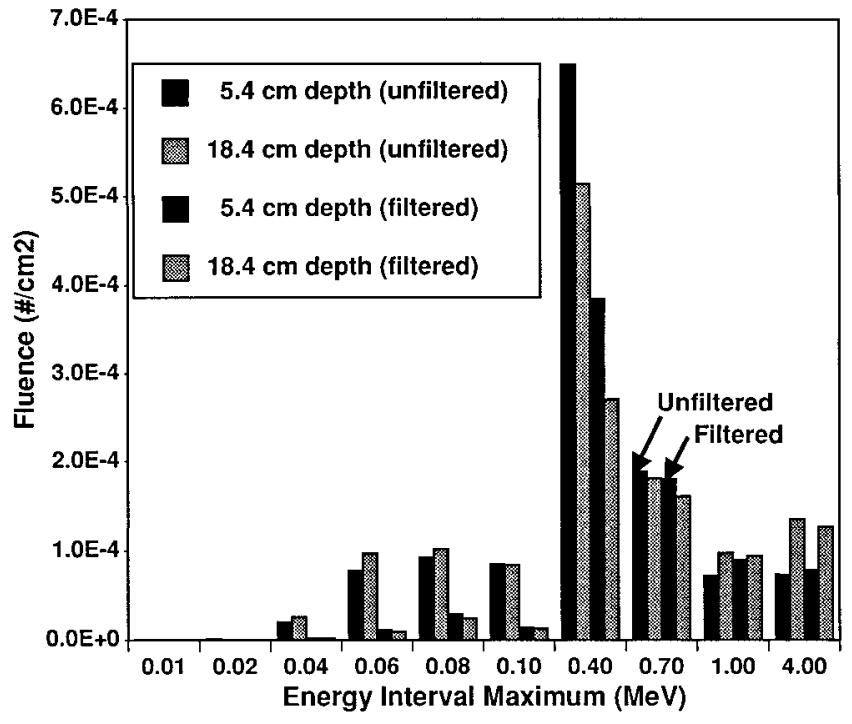

FIG. 2. The beam spectra within the phantom depend upon depth and field size. The two left-hand bars in each group represent the unfiltered beam at 5.4 and $18.4 \mathrm{~cm}$ depths along the central ray for the $25 \mathrm{~cm} \times 25 \mathrm{~cm}$ beam. The two right-hand bars in each group represent the filtered beam. At both depths, the number of photons in the higher energy intervals are unchanged while the photons below $400 \mathrm{keV}$ are effectively removed. 
single set of calibration films. The phrase "lateral scatter filtering" will be used to refer to the proposed method.

\section{MATERIALS AND METHODS}

The goal of a series of proof of concept experiments was to test whether lateral scatter filtering could effectively overcome the major obstacle to accuracy of high energy photon film dosimetry as described in Sec. I. Because film dosimetry includes other potential systematic errors such as film compression uniformity, processor effects, and densitometer or light source instability, it was important to minimize these problems and maximize the energy sensitivity of the film problem. For this reason, all experiments were performed at $4 \mathrm{MV}$, the lowest available linear accelerator energy in order to see maximal change in film response sensitivity with depth and field size. Each of the other known problems was addressed to minimize the film response sensitivity through methods to be described. The initial purpose was to demonstrate the efficacy of the method rather than to address the overall accuracy of photon film dosimetry or to produce a complete solution for the clinical physicist, although this is the goal for future studies.

\section{A. Ionization chamber reference measurements}

All experiments were performed with the $4 \mathrm{MV}$ linear accelerator (Varian Associates; Clinac 4), $80 \mathrm{~cm} \mathrm{SSD,} \mathrm{for}$ field sizes of $6 \mathrm{~cm} \times 6 \mathrm{~cm}$ and $25 \mathrm{~cm} \times 25 \mathrm{~cm}$. The $25 \mathrm{~cm} \times 25 \mathrm{~cm}$ size was the largest field width which could be measured with the 14-in.-wide film. Reference field calibration (cGy/monitor unit) and field size dependence at $d_{\max }$ in water were confirmed at the time of this study. In addition, percentage depth dose data, beam profiles at selected depths, as well as complete isodose curves for each beam were measured using an automated water phantom scanning system (Wellhofer, WP 600) with ionization chamber detectors (Wellhofer, Type IC-10) having an internal diameter of $6.0 \mathrm{~mm}$. These dose distributions served as the reference data to which all data measured in solid phantom materials and all film data were compared.

The calibration phantom used for these experiments consists of a set of $30 \mathrm{~cm} \times 30 \mathrm{~cm}$ polystyrene blocks of varying thickness, forming a total thickness of $40 \mathrm{~cm}$. The calibration phantom was used to verify that percentage depth dose was the same in polystyrene as in water. For this measurement the Markus ionization chamber (Nuclear Associates, PTW Model 30-329) was placed in a precisely machined opening in a slab of the phantom material. The phantom was positioned at $80 \mathrm{~cm}$ SSD on the $4 \mathrm{MV}$ accelerator and percentage depth dose was defined as follows:

$$
\mathrm{PDD}=\frac{\text { ionization }_{d}}{\text { ionization }_{d \max }} \times 100 \% \text {. }
$$

The actual dose to $d_{\max }$ in polystyrene was calculated by finding the field size dependence at $d_{\max }$ in the phantom and multiplying these relative numbers by $0.975 \mathrm{cGy} / \mathrm{MU}$, the calibrated dose rate for the $10 \mathrm{~cm} \times 10 \mathrm{~cm}$ field at $d_{\max }$.

\section{B. Film exposure techniques}

All dosimetric film studies were performed using ReadyPack film (Kodak, XV-2) processed with the $90 \mathrm{~s}$ automatic processor (Kodak, X-Omat) normally used for imaging within the clinical radiotherapy department. In order to minimize variations, each experiment and corresponding film calibration was performed using film from the same box.

\section{Densitometers}

Film densitometry has traditionally been performed using manual transmission densitometers or scanning transmission densitometers. Both of these methods are slow and have resolution limited to 1-2 $\mathrm{mm}$ because of the size of the aperture used. Newer devices using linear charge-coupled device (CCD) scanners or slow-scan, cooled CCD cameras are now commercially available as radiotherapy film densitometers. For these preliminary investigations, a cooled, solid state CCD camera system custom configured for film densitometry was selected because of its low noise and excellent linearity of response to light, as well as the high speed of data acquisition, high resolution, and geometric precision (Photometrics, Ltd., Tucson, AZ). ${ }^{8}$ The choice of densitometer type should not be critical to the outcome of this study so long as calibration films and experimental films are measured with the same device and the device has sufficient accuracy over the range of densities to be measured. For example, the measurement of low densities $(<0.30)$ with a densitometer which has a stated accuracy of \pm 0.01 density units may not produce reliable data since the uncertainty of measurement will be a large fraction of the expected result. For a net film density of $0.30 \pm 0.01$ the error is $3.3 \%$ and for a net density of $0.20 \pm 0.01$ it is $5 \%$. These values do not include the uncertainty associated with setting the density offset to automatically subtract film fog.

\section{Film phantom}

The polystyrene film phantom consists of a custom designed set of $40.6 \mathrm{~cm} \times 43.2 \mathrm{~cm}$ polystyrene blocks forming a total thickness of $35 \mathrm{~cm}$. This polystyrene is from the same production batch as the ion chamber calibration phantom. For all experimental films the accelerator beam was directed at the floor. For vertical film irradiation, with the film plane parallel to the beam central axis, the phantom was assembled and placed in an aluminum compression box with the top side open as shown in Fig. 1. For exposure perpendicular to the beam central axis the blocks were removed from the box and stacked on the patient support assembly of the accelerator. For this configuration the only compression was that provided by the weight of the blocks.

\section{Film orientation}

Since film density for horizontal exposures may not always match the density at the corresponding depths for films exposed vertically, it has been suggested that film orientation for calibration should be the same as film orientation for experimental exposure. ${ }^{3}$ Because this observation about film orientation was made originally using industrial type film, 
the investigation of this reported disparity was repeated for the $4 \mathrm{MV}$ x-ray beam with XV-2 film. Films were sandwiched between each of the stacked polystyrene blocks of the film phantom with the $35-\mathrm{cm}$-thick phantom in position for horizontal film exposure. The beam was perpendicular to the entrance surface of the phantom. The density at the center of each of these films was compared to the density at the corresponding depth along the center of a film which had been exposed vertically within the polystyrene phantom and with the film plane parallel to the beam direction. The experiment was performed for $6 \mathrm{~cm} \times 6 \mathrm{~cm}$ and $25 \mathrm{~cm} \times 25 \mathrm{~cm}$ fields at $80 \mathrm{~cm}$ SSD. No significant difference in densities was noted due to orientation. Consequently, all calibration film sets were exposed using the sandwiched horizontal film and stacked block configuration. This provided a quick and simple setup (no compression device) and facilitated subsequent digitization with the CCD densitometer.

\section{Film calibration procedure}

To measure the change in film sensitivity with field size and depth, and to relate film density to radiation dose, sets of calibration films were exposed for field sizes of $5 \mathrm{~cm} \times 5 \mathrm{~cm}, \quad 6 \mathrm{~cm} \times 6 \mathrm{~cm}, \quad 10 \mathrm{~cm} \times 10 \mathrm{~cm}$, and $25 \mathrm{~cm} \times 25 \mathrm{~cm}$ at a depth of $5 \mathrm{~cm}$ and for $6 \mathrm{~cm} \times 6 \mathrm{~cm}$ and $25 \mathrm{~cm} \times 25 \mathrm{~cm}$ at a depth of $15 \mathrm{~cm}$. Each calibration run consisted of a set of films positioned one at a time at the same location in the phantom and irradiated for various monitor unit (MU) settings from 5 to $90 \mathrm{MU}$ in steps of 5,10 , or 20 MU. An unexposed film was processed at the same time as the calibration films in order to determine the fog level due to the film emulsion. This density was subtracted from the density measured on each of the calibration films. Densities were measured with the cooled CCD camera using a $512 \times 512$ digitization matrix with 12 bit conversion resolution. The average central density values were based on the central $20 \times 20$ pixel values of the matrix.

As a result of an initial analysis of the data, the $6 \mathrm{~cm} \times 6 \mathrm{~cm}$ field at $5 \mathrm{~cm}$ depth, $75 \mathrm{~cm} \mathrm{SSD}$, was chosen as the reference setup for film calibration because further decreases in size or depth did not alter the dose versus density curve for this beam energy.

For each MU setting, the dose delivered to the stacked block phantom was measured with an ionization chamber and corrected for monitor unit end effects. The dose to each film position (depth) was then plotted as a function of net density and the resulting calibration graph was used to convert density to dose for an accompanying set of experimental films. Each set of experimental film data was accompanied by a set of calibration film measurements.

\section{Unmodified polystyrene phantom}

The unmodified polystyrene phantom used for these experiments has been described above. Films were placed between slabs of polystyrene held vertically in an aluminum compression box. The corner of the paper jacket was punctured using a thumb tack to release air in the film packet and to mark the film orientation. Approximately $5 \mathrm{~cm}$ of film was left above the phantom surface and was gently folded over and taped. For photon beam irradiation, this method produces a well-defined beam entrance line on the film. For each experimental setup the phantom was leveled and the SSD set to $80 \mathrm{~cm}$.

To determine the magnitude of the film sensitivity effect, films were exposed in the unmodified polystyrene phantom with film in the vertical orientation for comparison with ionization chamber depth dose data. Small and large field sizes were used to calculate isodose curves from the film measurements for comparison with ionization chamber isodose distributions.

\section{E. Modified polystyrene phantom}

A modification to the phantom was then designed to test whether a small thickness of high atomic number material could be sandwiched within the phantom to preferentially filter the low energy lateral scatter without significantly altering the actual tissue dose distribution in the central plane.

Lead foils were placed in the phantom parallel to the film and equidistant from the film plane on both sides (Fig. 1). The foils did not intercept the primary beam since the film plane corresponded to the central plane of the accelerator beam. Foils of thickness $0.15,0.30,0.46$, and $0.76 \mathrm{~mm}$ were investigated at distances of $0,0.6,1.2$, and $1.9 \mathrm{~cm}$ from the film to determine the optimal conditions needed to obtain acceptable agreement with ionization chamber depth dose data.

Using this modified phantom, depth dose, beam profiles, and isodose distributions were compared to those of the unmodified polystyrene phantom with no lead foils. In addition, depth dose was measured using an ionization chamber (Farmer type) in a calibration water phantom with lead foils positioned in the water phantom in a geometry similar to the film phantom geometry. In this way it was possible to determine the actual change in depth dose due to the lead foils but not related to film sensitivity.

\section{RESULTS}

\section{A. Polystyrene/water comparison}

Percentage depth doses measured using a parallel plate Markus type chamber in the polystyrene phantom for the $6 \mathrm{~cm} \times 6 \mathrm{~cm}$ and $25 \mathrm{~cm} \times 25 \mathrm{~cm}$ fields of a $4 \mathrm{MV}$ linear accelerator were compared to the percentage depth dose in water measured using a water phantom scanning system with ionization chamber detectors. For the two phantoms, the maximum variation from the mean for the two field sizes investigated was $\pm 1 \%$. It was concluded from this experiment that for the limited objectives of the present investigation polystyrene and water were equivalent for $4 \mathrm{MV}$ beam energy. Consequently, beam profiles and isodose distributions measured in the scanning water phantom could be used as isomorphic reference data for the polystyrene film phantom data without the necessity of geometrically scaling the film data. 


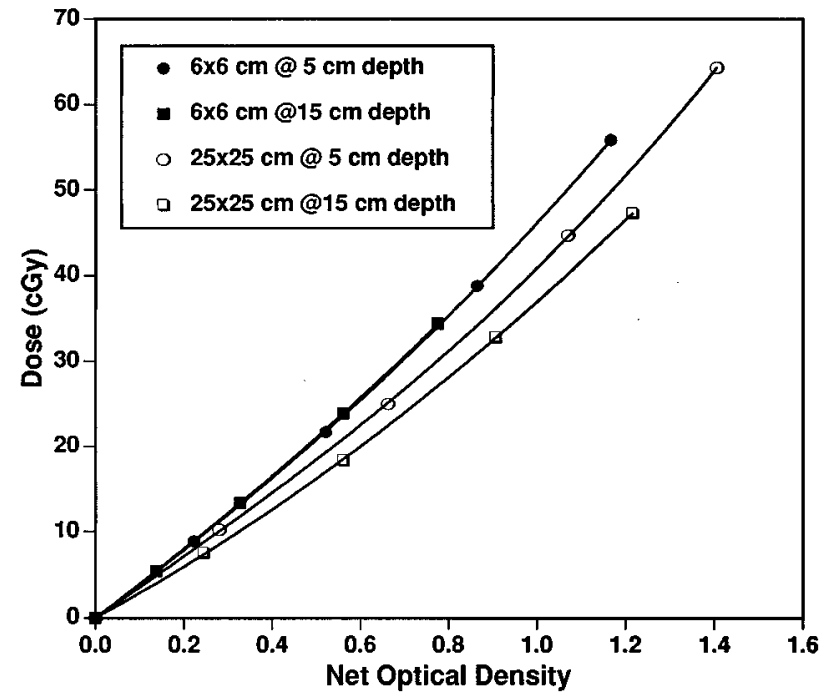

Fig. 3. Film sensitivity depends upon field size and upon depth, particularly for large field sizes. For sizes smaller than $10 \mathrm{~cm} \times 10 \mathrm{~cm}$ the change is small (not shown), and for sizes less than $6 \mathrm{~cm} \times 6 \mathrm{~cm}$ no change in sensitivity occurs. For small fields with less scatter the change in sensitivity with depth is not apparent.

\section{B. Dose versus density}

The results for the $6 \mathrm{~cm} \times 6 \mathrm{~cm}$ and $25 \mathrm{~cm} \times 25 \mathrm{~cm}$ fields shown in Fig. 3 emphasize the importance of the film sensitivity as a function of field size and depth. As the field size was increased the dose required to produce a given density on the film was reduced. Compare for example, data for the $6 \mathrm{~cm} \times 6 \mathrm{~cm}$ field shown in Fig. 3, which demonstrates little change in sensitivity for the 5 and $15 \mathrm{~cm}$ depths (Fig. 3), with the data for the $25 \mathrm{~cm} \times 25 \mathrm{~cm}$ field which is significantly different at 5 and $15 \mathrm{~cm}$ depths. For field sizes smaller than $10 \mathrm{~cm} \times 10 \mathrm{~cm}$ (not shown) there was little change in sensitivity, which would be expected given that there are significantly less low energy scattered photons in small fields. This change in sensitivity with field size and depth is the effect which has previously limited the use of film for photon beam dosimetry.

The variation of dose with field size at the $5 \mathrm{~cm}$ depth was measured using an ionization chamber and the values were normalized to the $6 \mathrm{~cm} \times 6 \mathrm{~cm}$ field and are presented in Fig. 4. Also shown are the relative doses based on the $6 \mathrm{~cm} \times 6 \mathrm{~cm}$ film density calibration data which demonstrates the need to have specific calibration data for each field size. The choice of $6 \mathrm{~cm} \times 6 \mathrm{~cm}$ at $5 \mathrm{~cm}$ depth for all calibration films is based on the fact that further reduction in field size or increase in depth did not affect the film sensitivity curve. It seems apparent that this set of conditions represents the film response to the higher primary beam energy with minimal film sensitivity enhancement that would result from the lower scatter energies.

A typical graph of dose versus density is shown in Fig. 5. Ideally this graph should be a straight line at low doses, but was found to be slightly nonlinear for this film type and CCD densitometer combination. For this study a simple fit to an equation of the form

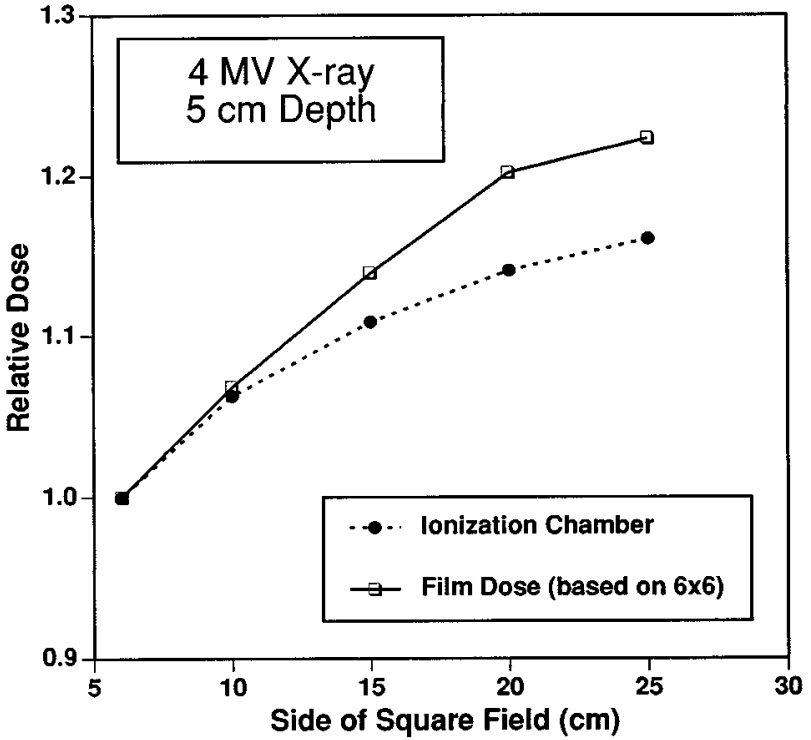

FIg. 4. Relative dose at $5 \mathrm{~cm}$ depth for a fixed monitor unit setting is shown as a function of field size. Film doses calculated based on $6 \mathrm{~cm} \times 6 \mathrm{~cm}$ calibration films overpredict the dose for larger sizes. Without lateral scatter filtering, calibration must be repeated for each range of field sizes to be measured. Lateral scatter filtering attempts to remove the cause of the increased response so that a single set of calibration films is sufficient.

$$
y=a^{*}\left(b x+c x^{2}+d x^{3}\right)
$$

yielded acceptable results for maximum doses of $80 \mathrm{cGy}$ or less. Values of $a, b, c$, and $d$ were found from an initial data set using polynomial curve fitting routines (Deltapoint, Inc., Deltagraph Professional). The initial values, $b=148.4$, $c=-1.73$, and $d=15.72$, were used as constants in all calibration data sets. Only the value of " $a$," the sensitivity mul-

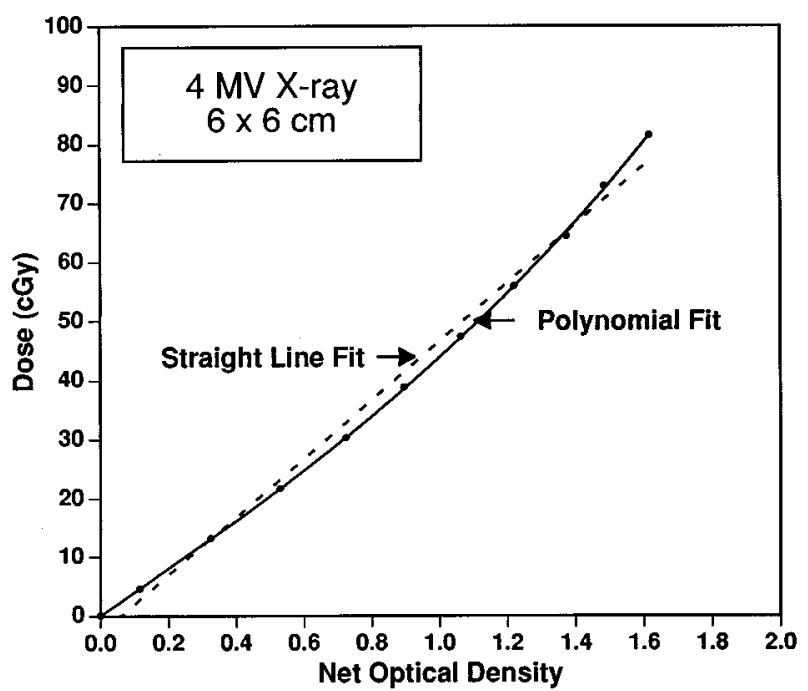

FIG. 5. The exact shape of the film response curve is a function of the combination of film and densitometry device. For the CCD camera and Kodak XV film used, a polynomial of the form $y=a\left(b x+c x^{2}+d x^{3}\right)$ was found to provide a significantly better fit than a simple straight line approximation. After the initial fit, the values of $b, c$, and $d$ were held constant and only the value of $a$, the sensitivity multiplier, was found to vary. 
TABLE I. Comparison of percentage depth dose measured using ionization chamber and film (4 MV). (Numbers in parentheses are ratios of present measurement to ion chamber reference.)

\begin{tabular}{cccccc}
\hline \hline & $\begin{array}{c}\text { Depth }(\mathrm{cm}) \\
\text { Ion chamber } \\
\text { (reference) }\end{array}$ & $\begin{array}{c}\text { Ion chamber } \\
\text { with foil }\end{array}$ & $\begin{array}{c}\text { Film } \\
\text { no foil }\end{array}$ & $\begin{array}{c}\text { Film } \\
\text { with foil }\end{array}$ \\
\hline $25 \times 25 \mathrm{~cm}$ & 5 & 84.3 & $83.9(1.00)$ & $94.3(1.12)$ & $85.7(1.02)$ \\
& 10 & 65.5 & $64.0(0.98)$ & $82.4(1.26)$ & $65.3(1.00)$ \\
& 15 & 49.9 & $47.8(0.96)$ & $69.6(1.39)$ & $49.2(0.99)$ \\
& 20 & 37.5 & $35.3(0.94)$ & $56.5(1.51)$ & $36.1(0.96)$ \\
& 25 & 28.1 & $26.1(0.93)$ & $45.1(1.60)$ & $27.0(0.96)$ \\
& 30 & 21.0 & & $34.6(1.65)$ & $20.1(0.96)$ \\
$6 \times 6 \mathrm{~cm}$ & 5 & & & $82.4(1.04)$ & $81.7(1.03)$ \\
& 10 & 56.3 & & $60.7(1.08)$ & $58.5(1.04)$ \\
& 15 & 39.5 & & $31.5(1.10)$ & $41.1(1.04)$ \\
& 20 & 27.7 & & $22.8(1.13)$ & $28.7(1.04)$ \\
& 25 & 19.5 & $19.1(0.98)$ & $16.0(1.16)$ & $14.4(1.04)$ \\
\hline \hline
\end{tabular}

tiplier, was found to vary for a given combination of film type, film densitometer, and photon beam energy. This variation of sensitivity is most likely related to processing conditions and variation in film sensitivity from batch to batch. It is important to note that the specific values of $a, b, c$, and $d$ may depend upon the particular film densitometry method used.

\section{Comparison of films from unmodified and modified polystyrene phantoms}

Films were exposed using the unmodified polystyrene film phantom and density values were converted to dose based on the $6 \mathrm{~cm} \times 6 \mathrm{~cm}$ calibration films. The resulting percentage depth dose curve calculated from film density was compared to the actual percentage depth dose measured with an ionization chamber in polystyrene and the results are presented in Table I. Relative to the ion chamber measurements, the film percentage depth doses are higher by $26 \%$ at $10 \mathrm{~cm}$ depth and by $65 \%$ at $30 \mathrm{~cm}$ depth for the $25 \mathrm{~cm} \times 25 \mathrm{~cm}$ field.

In order to address the over-response of film to very low energy photons, lead foils were placed in the phantom parallel to the film plane to filter the lateral scatter to the film (Fig. 1). The effect of foil to film separation distance and foil thickness were investigated in order to obtain a single optimum distance-thickness combination, and the results are presented in Figs. 6 and 7. In Figs. 6 and 7, the dose is calculated using data from the $6 \mathrm{~cm} \times 6 \mathrm{~cm}$ calibration films. Film/foil separation distances of $0,0.6,1.2$, and $1.9 \mathrm{~cm}$ and foil thicknesses of $0,0.15,0.30,0.46$, and $0.76 \mathrm{~mm}$ lead foil were included in the investigation. At $0 \mathrm{~cm}$ film/foil separation distance the curve shows the effect of electrons coming from the lead due to interactions within the foil. This intensification effect exaggerates the shape of the depth dose curve and the nonuniform film/foil contact is apparent in the data (Fig. 6). At $0.6 \mathrm{~cm}$ the scattered electrons are absorbed in the intervening polystyrene. Further increase in film/foil separation distance produced only minor changes in the calculated dose curve. Figure 7 shows the effect of changing foil thickness. A single thickness of $0.15 \mathrm{~mm}$ causes a sig- nificant decrease in the calculated dose with only subtle changes as additional layers are added. The best match to ion chamber percentage depth dose data was observed for lead foil thickness of $0.46 \mathrm{~mm}$ with a $1.2 \mathrm{~cm}$ film/foil separation distance.

In order to determine the effect of the lead foil on the actual percentage depth dose in water, measurements were made in a calibration water phantom using an ionization chamber. Lead foils attached to 10 -mm-thick polystyrene sheets were spaced $25 \mathrm{~mm}$ apart and placed in the water phantom to simulate the film measurement geometry. Due to the attenuation of the foil, dose decreased at $d_{\max }$ by $3.5 \%$. Percentage depth dose also decreased with the foils in place

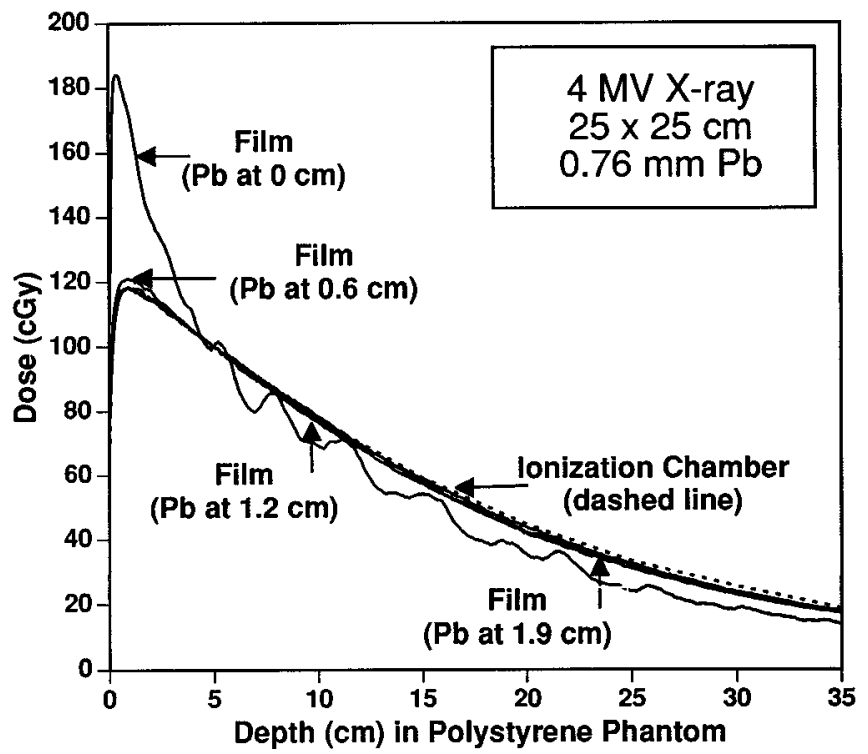

FIG. 6. Lead foils placed adjacent to the film show an exaggerated response at shallow depths and a wavy appearance due to undulations in their surfaces. At distances of $0.6,1.2$, and $1.9 \mathrm{~cm}$ the foils are beyond the range of most of the electrons set in motion within the foil, so that the lead acts only as a filter. Each curve is normalized to $5 \mathrm{~cm}$ depth to emphasize its overall shape relative to the ionization chamber curve. 


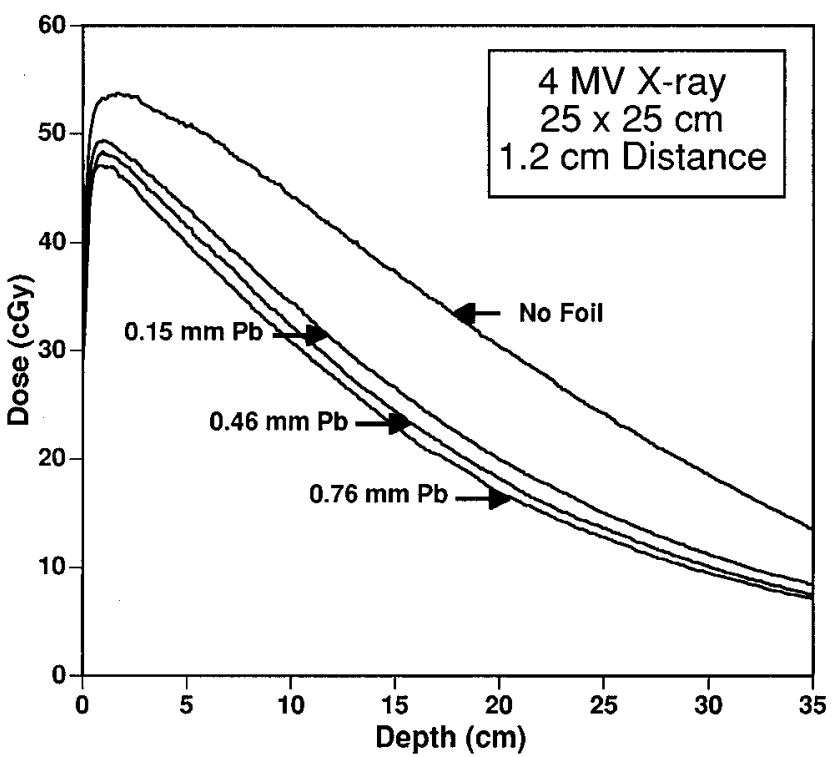

FIG. 7. The effect of foil thickness on the shape of the depth dose curve is shown. A thin lead foil $(0.15 \mathrm{~mm})$ dramatically reduces the over-response of the film. Additional thicknesses are added to produce the best depth dose match over the range of field sizes to be measured.

because the foils remove a larger fraction at increasing depths. This occurs because a greater proportion of the beam at depth is due to scatter within the phantom. The change in actual depth dose was much less than the change in film response, however, and the overall effect is summarized in Table I. Finally, graphs showing percentage depth dose for the ionization chamber and the film with and without lead are shown in Fig. 8. It is apparent that the improvement in accuracy of depth dose determination using film for the $25 \mathrm{~cm} \times 25 \mathrm{~cm}$ field is very significant. The improvement in the $6 \mathrm{~cm} \times 6 \mathrm{~cm}$ field measurement is significant, although not as dramatic. This is to be expected because the $6 \mathrm{~cm} \times 6 \mathrm{~cm}$ field has less low energy scatter to the central plane than the $25 \mathrm{~cm} \times 25 \mathrm{~cm}$ field, and hence less film overresponse before filtering.

Several authors have suggested that photon density distributions normalized to central axis ionization chamber data produce acceptable isodose distributions. 6,9 However, the beam spectrum also changes with distance from the central ray primarily because of changes in the proportion of scatter and differences in flattening filter thickness. A $5 \mathrm{~cm}$ depth profile for the $4 \mathrm{MV}, 25 \mathrm{~cm} \times 25 \mathrm{~cm}$ field, is shown in Fig. 9(a) for film in the unmodified phantom and for ion chamber. Ionization chamber off-axis ratios are higher than film ratios because the film response is maximum near the beam center where scatter is maximum. Since the profile is normalized to the central ray, the off-axis points seem lower than expected. The dose measured by the film just beyond the beam edge is also much higher than the ionization chamber because most of the radiation at this point is low energy scatter to which the film over-responds. The same profile from the modified film phantom is shown in Fig. 9(b) and demonstrates much better agreement with ionization chamber data within the
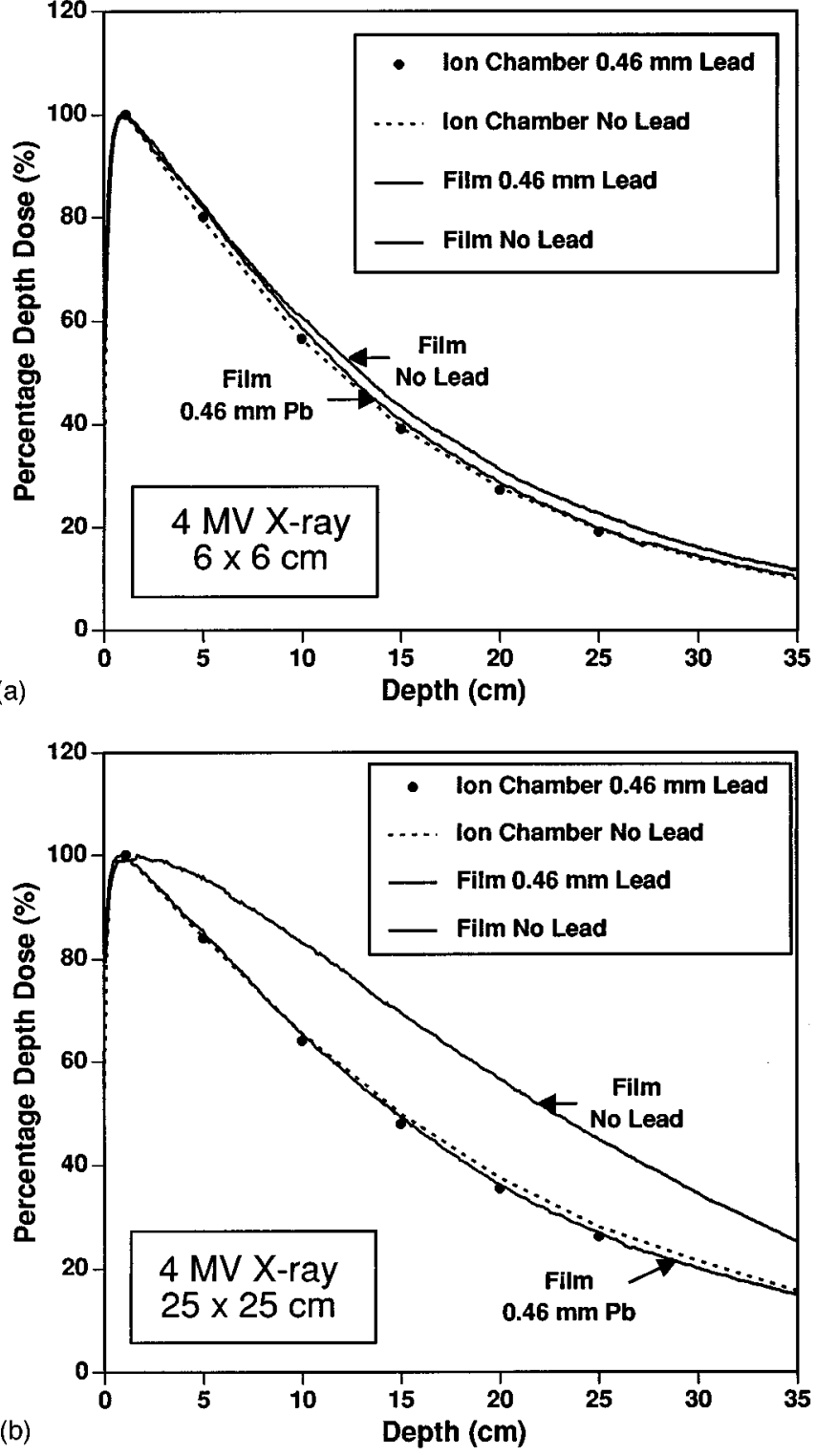

FIG. 8. The final comparison of depth dose data measured by ion chamber with and without foils and by film with and without foils is shown for (a) the $6 \mathrm{~cm} \times 6 \mathrm{~cm}$ field and (b) the $25 \mathrm{~cm} \times 25 \mathrm{~cm}$ field. The perturbation caused by the presence of the filters is small relative to the dramatic improvement in the film response.

primary beam and just beyond the beam edge. The effect at other depths is similar.

The calculation of isodose distributions based on film density measurement was the ultimate goal of this experiment. Figure 10 compares isodose distributions based on a film irradiated in the unmodified phantom and in the modified phantom. Calibration was based on $6 \mathrm{~cm} \times 6 \mathrm{~cm}$ dose versus density for both sets of data. The repeatability of the depth dose measurements for $4 \mathrm{MeV}, 80 \mathrm{~cm}$ SSD, $25 \mathrm{~cm} \times 25 \mathrm{~cm}$ field is summarized in Table II.

The improvement in accuracy using the modified film phantom was dramatic. The technique of filtering with high atomic number material to improve agreement between film 


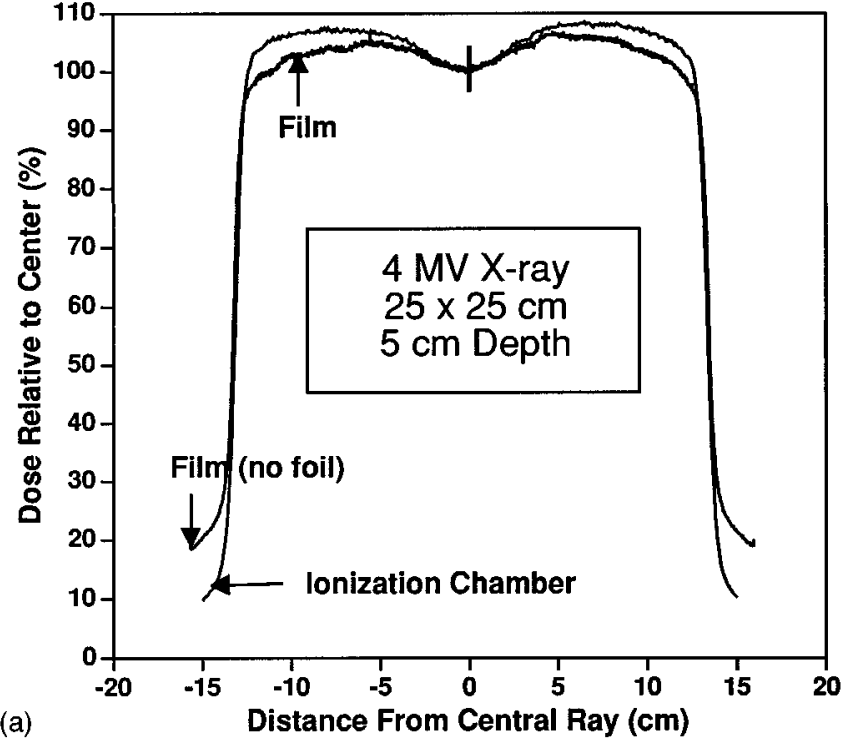

(a)

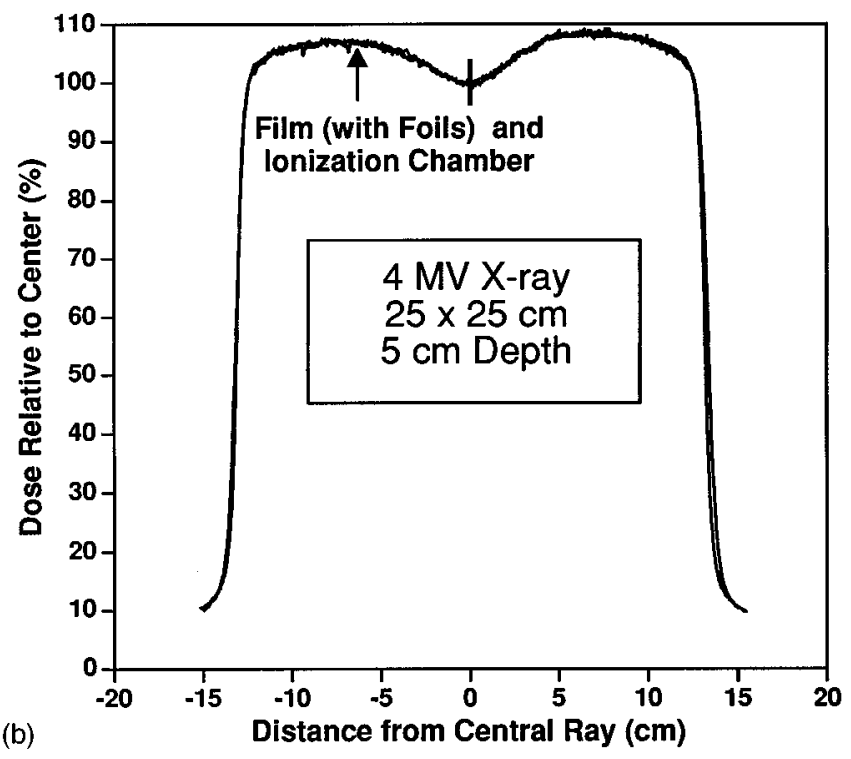

FIG. 9. (a) In this beam profile, measured at $5 \mathrm{~cm}$ depth with film, the beam appears flatter than it actually is. This is because the film over-responds to the greater proportion of scatter near the beam center and by normalizing to this point, the edges appear too low. The film also over-responds to the low energy scatter outside the beam edge in the penumbra region. (b) With lateral scatter filtering, the profile agrees well in all regions with the ionization chamber data.

and ionization chamber measurements produced very encouraging results.

\section{CONCLUSIONS}

The use of film for measurement of photon beam dose distributions within tissue equivalent phantoms was investigated. Relative to ion chamber data, differences of up to $65 \%$ in percentage depth dose measurement were observed in the polystyrene film phantom at $4 \mathrm{MV}$ for the $25 \mathrm{~cm} \times 25 \mathrm{~cm}$ field. The film density to dose conversion was based on a calibration field size of $6 \mathrm{~cm} \times 6 \mathrm{~cm}$. This discrepancy is due to the over-response of film to low energy scattered photons within the phantom. By filtering the low energy photons

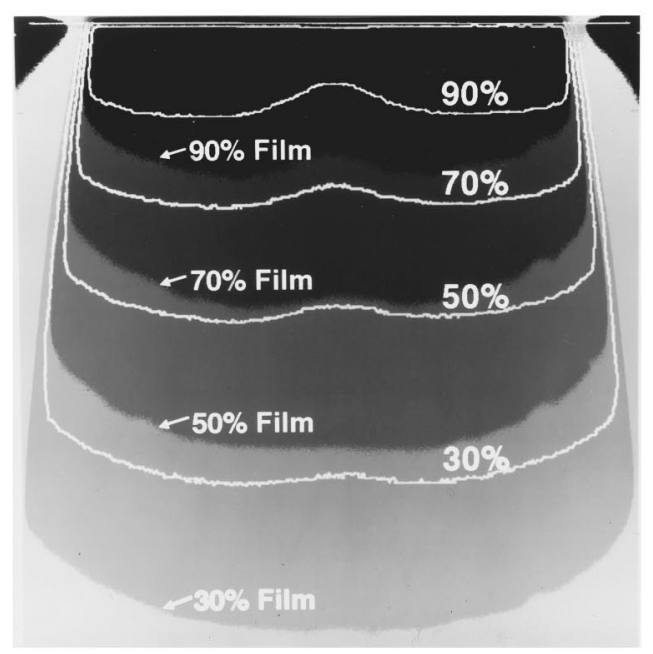

(a)

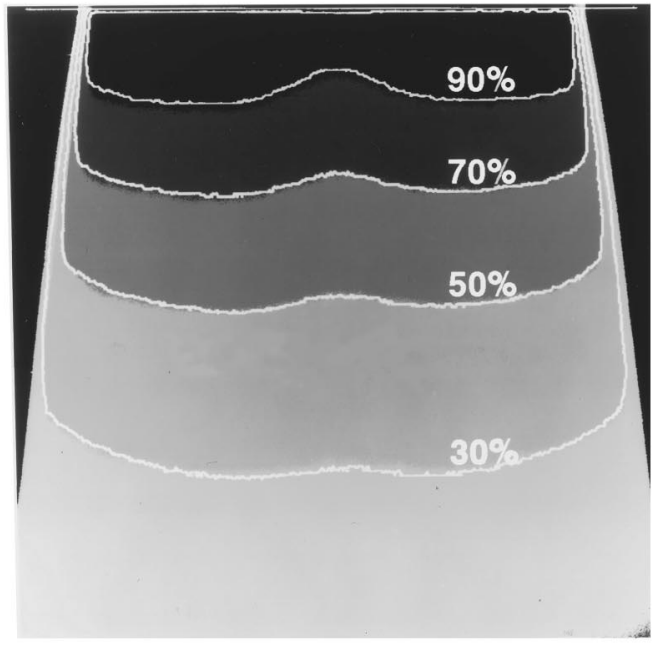

(b)

FIG. 10. Greyscale isodose curves are shown for film as calculated using the single $6 \mathrm{~cm} \times 6 \mathrm{~cm}$ calibration film set for density to dose conversion. The white lines overlaid are ionization chamber curves measured for this beam. (a) Results without lateral scatter filtering show large differences in depth dose between film and ion chamber. (b) With lateral scatter filtering excellent agreement is obtained.

from the beam with lead foils parallel to the film plane, significant improvements were made in the accuracy of film densitometry in photon beam dosimetry. A study of small $(6 \mathrm{~cm} \times 6 \mathrm{~cm})$ and large $(25 \mathrm{~cm} \times 25 \mathrm{~cm})$ field depth dose and beam profiles was performed for $4 \mathrm{MV} x$ rays. The

TABLE II. Repeatability of percentage depth dose measurement using film.

\begin{tabular}{cccc}
\hline \hline Depth $(\mathrm{cm})$ & Set 1 & Set 2 & Ratio set 1/set 2 \\
\hline 5 & 85.6 & 84.4 & 1.014 \\
10 & 65.3 & 65.0 & 1.005 \\
15 & 49.0 & 49.0 & 1.000 \\
20 & 36.3 & 36.2 & 1.003 \\
25 & 27.0 & 27.2 & 0.993 \\
30 & 20.1 & 20.2 & 0.995 \\
\hline \hline
\end{tabular}


maximum percent difference relative to ion chamber measurement was reduced to $4 \%$. Additional studies are required to demonstrate applicability of the method to the complete range of field sizes of interest.

Although the presence of lead foils in the phantom does perturb the depth dose, the error produced by this modification is balanced by the dramatic improvement in isodose curve agreement at $4 \mathrm{MV}$. Future studies using the same phantom for 6 and $18 \mathrm{MV}$ will further investigate the optimal lead thickness and distance for these energies. For higher energies the problem of film over-response is less severe, and the addition of the foils causes less perturbation of the depth dose, so some improvement is likely.

Future studies will also focus on interpretation of these experimental results using Monte Carlo simulation and extension of the concept to other film orientations. Presently the technique is limited to beam orientation with the central axis parallel to the film plane.
${ }^{1}$ R. Loevinger, C. J. Karzmark, and M. Weissbluth, "Radiation therapy with higher energy electrons. 1. physical considerations, 10 to $60 \mathrm{MeV}$," Radiology 77, 906-927 (1961).

${ }^{2} J$. Greening, "The photographic action of x-rays," Proc. Phys. Soc. London, Sec. B 64, 977-992 (1951).

${ }^{3}$ J. F. Williamson, "Film dosimetry of megavoltage photon beams: A practical method of isodensity-to-isodose curve conversion," Med. Phys. 8, 94-98 (1981).

${ }^{4}$ D. W. Anderson and F. St. George, "Comparison of film and ion chamber systems for depth-dose measurements for a 25 MV beam," Phys. Med. Biol. 24, 636-638 (1979).

${ }^{5} \mathrm{M}$. Ehrlich, Photographic Dosimetry of X- and Gamma Rays (U.S. Department of Commerce, Washington, DC, 1954).

${ }^{6}$ J. Purdy, "Instrumentation for dosimetric measurements," Proceedings of the AAPM Conference on Electron Linear Accelerators in Radiation Therapy, March 1978 (unpublished).

${ }^{7}$ J. A. Halbleib, R. P. Kensek, T. A. Mehlhorn, and G. D. Valdez, "ITs 3.0 integrated tiger series of coupled electron/photon Monte Carlo transport codes system," Report No. SAND91-1634, Sandia National Laboratory, 1992.

${ }^{8}$ J. H. Trueblood, S. E. Burch, K. J. Kearfott, and K. W. Brooks, "Radiographic Film Digitization," Digital Imaging: Proceedings of the 1993 AAPM Summer School (Medical Physics Publishing, Madison, WI, 1993). ${ }^{9}$ R. C. Granke, K. A. Wright, W. W. Evans, J. E. Nelson, and J. G. Trump, "The film method of tissue dose studies with $2.0 \mathrm{MeV}$ roentgen rays," Am. J. Roentgenol., Radium Ther. Nucl. Med. 72, 302-307 (1954). 